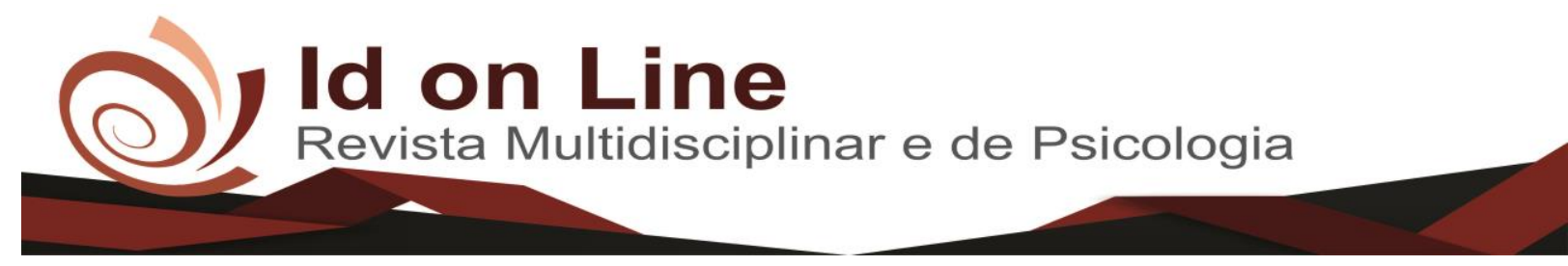

Artigo de Revisão

\title{
Acolhimento em Saúde Mental na Atenção Primária à Saúde: Revisão Integrativa
}

\author{
José Adelmo da Silva Filho; Adriana de Moraes Bezerra.
}

\begin{abstract}
Resumo: Objetivou-se compreender acerca do acolhimento em saúde mental na atenção primária à saúde - APS e elucidar as contribuições dessa tecnologia nas práticas de cuidar em saúde mental na APS. Trata-se de uma revisão integrativa da literatura, em que a busca foi realizada na LILACS, BDENF, MEDLINE e SciELO, com o cruzamento dos descritores acolhimento, saúde mental, atenção primária à saúde, atenção básica com o operador boolenao AND. Após a seleção dos estudos por meio dos critérios de exclusão e inclusão e, posteriormente, leitura na íntegra dos artigos, elencou-se categorias temáticas para melhor discussão dos resultados encontrados. Estas são: Fundamentalidade do Acolhimento, o acolher e o encaminhar: implicações do cuidado e, Barreiras encontradas no acolhimento em saúde mental na APS. Conclui-se que o acolhimento em saúde mental na atenção primária à saúde é fundamental para uma assistência pautada na integralidade do cuidado, em especial ao usuário que sofre de problemas psíquicos.
\end{abstract}

Descritores: Acolhimento; Saúde mental; Atenção primária à saúde, Atenção básica.

\section{Mental Health Hospital in Primary Health Care: an Integrative Review}

The objective was to understand about the reception in mental health in primary health care - PHC and to elucidate the contributions of this technology in PHC mental health care practices. It is an integrative review of the literature, in which the search was performed in LILACS, BDENF, MEDLINE and SciELO, with the cross-referencing of host, mental health, primary health care, basic care with the boolenao AND operator. After selecting the studies by means of the exclusion and inclusion criteria and, afterwards, reading the articles in their entirety, thematic categories were presented for a better discussion of the results found. These are: Fundamentality of the Reception, the welcoming and the sending: implications of the care and, Barriers found in the reception in mental health in the APS. It is concluded that the reception in mental health in primary health care is fundamental for a care based on integral care, especially the user who suffers from psychic problems.

Descriptores: Reception; Mental health; Primary health care, Primary care.

\footnotetext{
${ }^{1}$ Enfermeiro. Especialista em Saúde Mental. Residente em Saúde Mental Coletiva pela Escola de Saúde Pública do Ceará. adelmof12@gmail.com;

${ }^{2}$ Enfermeira. Mestra em Enfermagem pela Universidade Regional do Cariri. Docente do Departamento de Enfermagem da Universidade Regional do Cariri - URCA. Membro do Grupo de Pesquisa Tecnologias em Saúde no SUS. Membro do Núcleo de Avaliação de Tecnologias em Saúde (NATS) e Núcleo de Evidências da Universidade Regional do Cariri.

E-mail: adriana1mb@hotmail.com.
} 


\section{Introdução}

A Conferência Internacional sobre Cuidados Primários de Saúde realizada no ano de 1978, em Alma-Ata, consagrou a Atenção Primária à Saúde (APS) como a porta de entrada dos sistemas de saúde e o principal elemento de um sistema de saúde, sendo o primeiro nível para um processo contínuo de atenção à saúde (RODRIGUES et al., 2014). É a APS que deve deter a coordenação dos fluxos do usuário entre os serviços de saúde, na perspectiva de garantir o acesso do usuário aos serviços de saúde conforme sua demanda (GIOVANELLA; MENDONÇA, 2012).

O Brasil, por meio da Política Nacional de Atenção Básica, aprovada através da portaria No 2.436 de 21 de setembro de 2017, estabelece atribuições a todos os profissionais da Atenção Primária à Saúde, das quais estão: realizar o cuidado integral à saúde da população; realizar ações de promoção, proteção e recuperação da saúde e prevenção de doenças e agravos; participar do acolhimento dos usuários, proporcionando atendimento humanizado, realizando classificação de risco, identificando as necessidades de intervenções de cuidado, responsabilizando-se pela continuidade da atenção e viabilizando o estabelecimento do vínculo (BRASIL, 2017).

Nessa perspectiva, o acolhimento deve ser entendido como norteador transversal da atenção à saúde para garantir a integralidade do sujeito, e que para isto, os gestores do sistema sanitário devem assegurar um planejamento e execução de um projeto ético-político de gestão com vista a essa integralidade (RODRIGUES; BROGNOLI, 2014).

Reparando o processo de reforma psiquiátrica é possível perceber que a busca por implementações de serviços de saúde mental comunitários e territoriais antecedem a criação do SUS, e que as diretrizes implementadas com a criação do SUS, em especial a descentralização dos serviços de saúde, também redimensiona o acolhimento em saúde mental para além dos Centros de Atenção Psicossocial (CAPS) (RODRIGUES; BROGNOLI, 2014).

$\mathrm{O}$ acolhimento representa um momento de encontro vivo em ato, enquanto trabalho em saúde, mediado pela escuta qualificada, visando também, o estabelecimento de vínculo. Para tanto, acarreta compromisso e disponibilidade do profissional, como também do usuário, para a realização com efetividade (SILVA; ROMANO, 2015; FILHO, 2016).

Rodrigues e Brognnoli (2014) trazem o acolhimento em três dimensões singulares: Técnica, Postura e Reorientação do Serviço. Na dimensão Técnica do acolhimento utiliza-se do 
conhecimento e saber científico do profissional acolhedor, para que seja possível compreender e interpretar a demanda do usuário. A dimensão Postura compreende a forma que o profissional ou a equipe abordam o sujeito/acompanhante/família quando chegam no serviço e a dimensão Reorientação do Serviço traz o acolhimento como princípio de organizador do processo de trabalho.

Na saúde mental, segundo a Organização Pan-Americana de Saúde, o número de pessoas no mundo com algum transtorno mental, comportamental, neurobiológico ou psicossocial em 2001 seria aproximadamente 450 milhões de pessoas. A saúde mental vem ganhando espaço nas políticas públicas por compreender que as pessoas portadoras de algum sofrimento psíquico têm suas atividades cotidianas e relacionais prejudicadas, consequências também intrínsecas ao fato dessas pessoas vivenciarem o preconceito, exclusão, e o estigma que historicamente a saúde mental carrega (WHO, 2001; SOARES et al., 2015).

Sabendo-se que a APS é a principal porta de entrada do sistema de saúde, espera-se que demandas de saúde mental também sejam resolvidas pelos profissionais da APS, e se necessário, realizar encaminhamento para unidade especializada. Para que isto aconteça, é preciso que o acolhimento seja feito com maior cautela, justamente por entender que pacientes com alguma demanda de saúde mental possa manifestar distorções cognitivas, sendo crucial à atenção ao acolhimento para que seja possível realizar devolutiva mais adequada ao paciente (GARCIA et al., 2014).

Dessa forma, o acolhimento como dispositivo do processo de trabalho tem potencial para viabilizar o cuidado integral ao usuário, em busca da humanização na assistência. Para tanto, é imprescindível que os profissionais entendam a real importância do acolhimento, descaracterizando a visão de recepção, triagem e realização de encaminhamento sem adequada avaliação (JORGE et al., 2011).

Assim, definiu-se a questão que norteia o estudo: Qual a produção de conhecimento sobre acolhimento em saúde mental na atenção primária à saúde, no período de 2010 a 2017 ?

Objetivou-se compreender, por meio da literatura científica, acerca do acolhimento em saúde mental na atenção primária à saúde e elucidar as contribuições dessa tecnologia nas práticas de cuidar em saúde mental na APS. 


\section{Método}

O estudo ora elaborado caracteriza-se como uma revisão integrativa da literatura, definido como um método de pesquisa que tem como objetivo obter informações de determinado assunto, por meio da busca, avaliação crítica e síntese do estado do conhecimento pesquisado (SOARES et al., 2014).

Para realização, seguiu-se as seis etapas utilizadas na elaboração desse tipo de revisão, sendo: identificação do tema e elaboração da pergunta norteadora, estabelecimento de critérios para inclusão e exclusão de estudos, definição das informações a serem extraídas dos estudos selecionados, interpretação/discussão dos resultados, apresentação da revisão/síntese do conhecimento (MENDES; SILVEIRA; GALVAO, 2008).

Desse modo, a pergunta norteadora do estudo foi: Qual a produção de conhecimento sobre acolhimento em saúde mental na atenção primária à saúde, no período 2010-2017?

$\mathrm{O}$ estudo foi desenvolvido por meio de quatro bases de dados indexadas: Literatura Latino-Americana e do Caribe em Ciências da Saúde (LILACS), Base de dados de enfermagem (BDENF), Literatura Internacional em Ciências da Saúde (MEDLINE) e Scientific Electronic Library Online (SCIELO). A escolha das bases de dados justifica-se pela relevância científica apresentada nas produções dos diversos âmbitos da saúde.

O levantamento dos dados ocorreu por meio dos termos estabelecidos pelos Descritores em Ciências da Saúde (DeCS): acolhimento, saúde mental, atenção primária à saúde, atenção básica. Os descritores foram cruzados utilizando o operador booleano AND da seguinte forma: "acolhimento $A N D$ saúde mental", "acolhimento $A N D$ atenção primária à saúde", "acolhimento AND atenção básica”.

Optou-se por considerar como critérios de inclusão os estudos disponíveis na íntegra, gratuitos e em português, que fossem do tipo artigo e publicados no período de 2010 a 2017. Os critérios de exclusão dos estudos foram: duplicatas, estudos que não abordassem a temática do objetivo da revisão e estudos de revisão.

$\mathrm{Na}$ busca inicial nas bases de dados conforme descritores estabelecidos, foram encontrados na LILACS 810 resultados; na BDENF 237; na MEDLINE 26 e na SCIELO 141 estudos. Após aplicar os critérios de inclusão, restaram 413 artigos na LILACS; 126 na BDENF; 21 na MEDLINE e na SCIELO 101 artigos. Destes, após empregar os critérios de exclusão, 
restaram 4 artigos que compuseram a amostra do estudo, conforme vislumbrado na Tabela 01 e 02 . A busca foi realizada no mês de dezembro de 2017 .

Tabela 01 - Distribuição dos artigos publicados entre 2010 - 2017 conforme cruzamento dos descritores, Iguatu/CE, 2018.

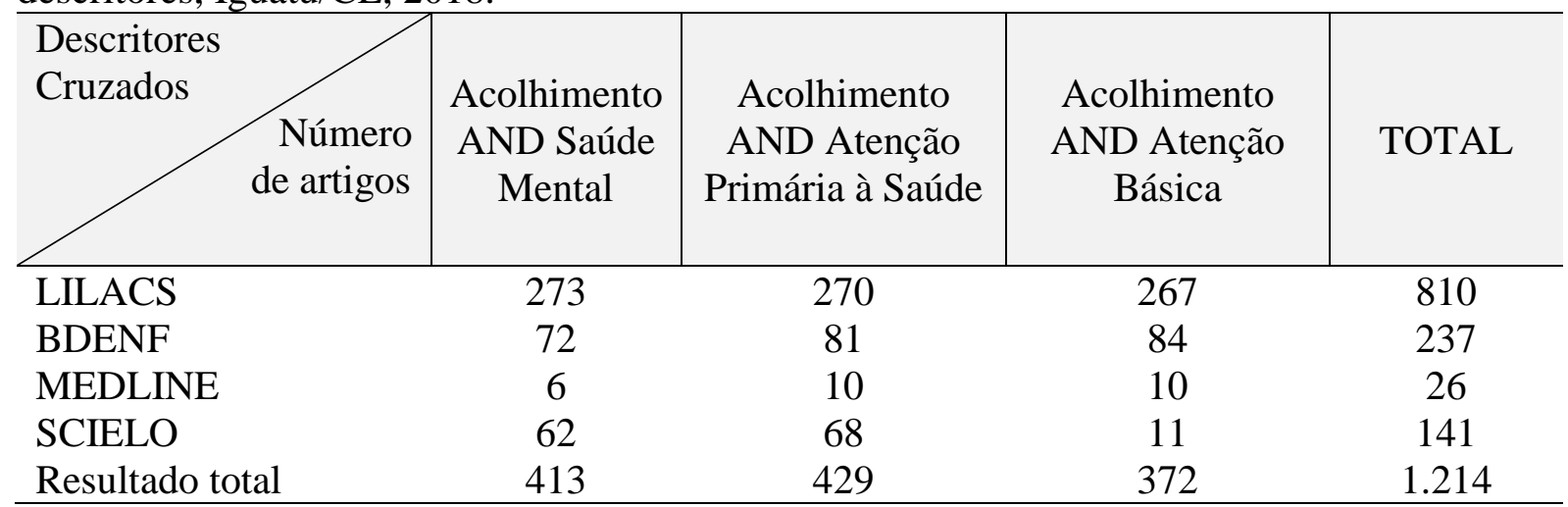

Fonte: elaborado pelo autor.

Tabela 02 - Distribuição dos artigos publicados entre 2010 - 2017 conforme critérios de inclusão e exclusão, Iguatu/CE, 2018.

\begin{tabular}{|c|c|c|c|c|c|}
\hline $\begin{array}{l}\text { Critérios de } \\
\text { Inclusão e } \\
\text { Exclusão Número } \\
\text { de artigos }\end{array}$ & LILACS & BDENF & MEDLINE & SCIELO & Total \\
\hline Resultado total & 810 & 237 & 26 & 141 & 1.214 \\
\hline $\begin{array}{l}\text { Após aplicar os } \\
\text { critérios de } \\
\text { inclusão }\end{array}$ & 413 & 126 & 21 & 101 & 661 \\
\hline $\begin{array}{l}\text { Após aplicar os } \\
\text { critérios de } \\
\text { exclusão }\end{array}$ & 2 & 1 & 0 & 1 & 4 \\
\hline Amostra final & & & & & 4 \\
\hline
\end{tabular}

Fonte: Elaborado pelo autor.

Foi utilizado um instrumento de coleta de dados para obter síntese do material selecionado, contendo título do artigo, autores, objetivos, tipo de estudo, base de dados indexado, periódico e ano, que resultou na construção de um quadro sinóptico (QUADRO 01).

Utilizou-se a análise descritiva dos resultados para possibilitar melhor compreensão do leitor. Por ser uma pesquisa de dados secundários por meio de bases de domínio público, dispensou-se a autorização do Comitê de Ética em Pesquisa com Seres Humanos (CEP). 


\section{Resultados e Discussão}

Os artigos selecionados mediante critérios pré-estabelecidos foram agrupados no Quadro 01 para melhor visualização.

Quadro 01 - Panorama geral dos artigos selecionados que abordavam o acolhimento em saúde mental na atenção primária à saúde, no período 2010-2017. Iguatu/CE, 2018.

\begin{tabular}{|c|c|c|c|c|c|c|}
\hline AUTORES & $\begin{array}{c}\text { TÍTULO DO } \\
\text { ARTIGO }\end{array}$ & OBJETIVOS & $\begin{array}{l}\text { TIPO DE } \\
\text { ESTUDO }\end{array}$ & INDEXADO & PERIODICO & ANO \\
\hline $\begin{array}{c}\text { Mielke FB, } \\
\text { Olschowsky } \\
\text { A }\end{array}$ & $\begin{array}{c}\text { AÇÕES DE } \\
\text { SAÚDE MENTAL } \\
\text { NA } \\
\text { ESTRATÉGIA } \\
\text { SAÚDE DA } \\
\text { FAMÍLIA E AS } \\
\text { TECNOLOGIAS } \\
\text { EM SAÚDE }\end{array}$ & $\begin{array}{c}\text { avaliar as } \\
\text { tecnologias em } \\
\text { saúde utilizadas } \\
\text { por equipes da } \\
\text { Estratégia Saúde } \\
\text { da Família para o } \\
\text { desenvolvimento } \\
\text { de ações de saúde } \\
\text { mental no } \\
\text { território }\end{array}$ & Qualitativo & SCIELO & $\begin{array}{l}\text { Esc. Anna } \\
\text { Nery }\end{array}$ & 2011 \\
\hline $\begin{array}{c}\text { Sucigan } \\
\text { DHI, } \\
\text { Toledo VP, } \\
\text { Garcia } \\
\text { APRF }\end{array}$ & $\begin{array}{c}\text { ACOLHIMENTO } \\
\text { E SAÚDE } \\
\text { MENTAL: } \\
\text { DESAFIO } \\
\text { PROFISSIONAL } \\
\text { NA } \\
\text { ESTRATÉGIA } \\
\text { SAÚDE DA } \\
\text { FAMÍLIA }\end{array}$ & $\begin{array}{l}\text { Compreender } \\
\text { como os } \\
\text { profissionais de } \\
\text { enfermagem de } \\
\text { equipes de saúde } \\
\text { da família } \\
\text { realizam o } \\
\text { acolhimento dos } \\
\text { pacientes de } \\
\text { saúde mental e } \\
\text { seus sentimentos } \\
\text { diante deste } \\
\text { trabalho }\end{array}$ & Qualitativo & BDENF & $\begin{array}{l}\text { Revista da } \\
\text { rede de } \\
\text { Enfermagem } \\
\text { do Nordeste } \\
\text { (Rev. Rene) }\end{array}$ & 2012 \\
\hline $\begin{array}{l}\text { Minóia NP, } \\
\text { Minozzo F }\end{array}$ & $\begin{array}{l}\text { Acolhimento em } \\
\text { Saúde Mental: } \\
\text { Operando } \\
\text { Mudanças na } \\
\text { Atenção Primária à } \\
\text { Saúde }\end{array}$ & $\begin{array}{c}\text { Descrever o } \\
\text { processo de } \\
\text { implantação do } \\
\text { acolhimento às } \\
\text { pessoas em } \\
\text { sofrimento } \\
\text { psíquico em uma } \\
\text { unidade de APS, } \\
\text { no município de } \\
\text { Porto Alegre/RS }\end{array}$ & $\begin{array}{c}\text { Relato de } \\
\text { experiência }\end{array}$ & LILACS & $\begin{array}{c}\text { Revista } \\
\text { Psicologia: } \\
\text { Ciência e } \\
\text { Profissão }\end{array}$ & 2015 \\
\hline $\begin{array}{l}\text { Araujo ak, } \\
\text { Tanaka oy }\end{array}$ & $\begin{array}{l}\text { Avaliação do } \\
\text { processo de } \\
\text { acolhimento em } \\
\text { Saúde Mental na } \\
\text { região centro-oeste } \\
\text { do município de } \\
\text { São Paulo: a } \\
\text { relação entre } \\
\text { CAPS e UBS em } \\
\text { análise }\end{array}$ & $\begin{array}{l}\text { Entender o } \\
\text { acolhimento, } \\
\text { considerando a } \\
\text { percepção dos } \\
\text { trabalhadores e } \\
\text { identificando o } \\
\text { vínculo e a } \\
\text { articulação da } \\
\text { rede nesse } \\
\text { processo }\end{array}$ & $\begin{array}{l}\text { Hermenêutica } \\
\text { filosófica }\end{array}$ & LILACS & $\begin{array}{c}\text { Interface - } \\
\text { Comunicação, } \\
\text { Saúde, } \\
\text { Educação }\end{array}$ & 2012 \\
\hline
\end{tabular}

Fonte: elaborado pelo autor. Iguatu/CE, 2018. 
Dentre os quatros artigos selecionados, dois estavam indexados na LILACS, um na SCIELO e um na BDENF, a base de dados MEDLINE não obteve amostra que contemplasse os critérios deste estudo.

Quanto aos periódicos, os artigos estão distribuídos na Interface - Comunicação, Saúde, Educação, Revista Psicologia: Ciência e Profissão, Revista da rede de Enfermagem do Nordeste (Rev. Rene) e Esc. Anna Nery. Em relação ao ano de publicação, observa-se que, um foi publicado em 2011 e dois em 2012 e apenas um artigo no ano de 2015, evidenciando que a temática ainda é pouco investigada e indexada nessas bases de dados.

No que se refere ao delineamento dos estudos, os artigos são de abordagens variadas, tendo destaque dois artigos de abordagem qualitativa, um relato de experiência e um estudo de base hermenêutica filosófica, todos desenvolvidos no Brasil.

Após a leitura na íntegra dos artigos que compuseram a amostra do estudo, elencou-se categorias temáticas para melhor discussão dos resultados encontrados. Estas são: Fundamentalidade do Acolhimento, o acolher e o encaminhar: implicações do cuidado e, Barreiras encontradas no acolhimento em saúde mental na APS.

\section{CATEGORIA 1: Fundamentalidade do Acolhimento}

A percepção do acolhimento, enquanto conceito-ferramenta no processo de trabalho, tem potencial para implicar na produção de cuidados terapêuticos no usuário que se encontra em sofrimento psíquico e que por meio do espaço propiciado no acolher seja possível surgir as demandas do sujeito (SCHEIBELB; FERREIRA, 2011).

Essencial para um atendimento humanizado, o acolhimento ao doente mental na APS se torna um eixo norteador da assistência, principalmente por ser um diferencial na condução das intervenções realizadas ao sujeito que se encontra em sofrimento (CORREIA; BARROS; COLVERO, 2011).

O artigo de Sucigan, Toledo e Garcia (2012) referem que os profissionais da Atenção Primária à Saúde devem ser capacitados para fazer um acolhimento com qualidade ao sujeito com sofrimento psíquico, por compreenderem que a realização do acolhimento qualificado favorece ao usuário vivenciar sua subjetividade, instigando a reflexão sobre o seu mal-estar. Referem ainda que a prática do acolhimento qualificado minimiza a prevalência do modelo 
biomédico, tornando transversal o cuidado terapêutico e contribuindo para desmedicalização do sujeito.

A percepção de Mielke e Olschowsky (2011) traz a compreensão do acolhimento como uma prática que possibilita a universalização do acesso, desde que todos sejam acolhidos pelo serviço, e que tem suas ações centralizadas no sujeito, tendo como pilares nas relações entre o profissional e o usuário a humanização, solidariedade e cidadania.

$\mathrm{O}$ acolhimento se torna determinante no processo de cuidar dentro da saúde mental na medida que transpassa os conceitos de patologia e diagnóstico da doença mental, deixando sobressair o cuidado integral alicerçado na individualidade e singularidade de cada indivíduo, sobretudo quando concebido na prática assistencial como uma ferramenta de diálogo entre os sujeitos envolvidos (JORGE et al., 2011).

A atenção Primária à Saúde por ser a ordenadora da Rede de Atenção à Saúde (RAS) e a coordenadora do cuidado, se torna o primeiro nível de atenção ligado diretamente aos usuários com demandas de saúde mental, e devido essa característica impar é de fundamental importância que o acolhimento seja fortalecido dentro desse âmbito de cuidados, tanto para mobilizar a sensibilidade dos profissionais através de ação reflexiva, ética e solidária no momento do acolhimento, quanto por contribuir com o apoio social e medidas de combate ao estigma e preconceito que circundam o doente mental, dessa forma, fortalecendo os princípios do SUS no que se refere a integralidade e equidade entre os sujeitos (SANTOS; SANTOS, 2011).

\section{CATEGORIA 2 : $O$ acolher o o encaminhar: implicações do cuidado}

Tradicionalmente, o acolhimento no campo da saúde tem sido caracterizado ora como uma dimensão espacial, ora como uma ação de triagem e encaminhamento, restringindo-se às ações pontuais, isoladas e descomprometidas com a produção de responsabilização e vínculo com a clientela. Em suas várias definições, o acolhimento como ato de acolher expressa uma ação de aproximação com o outro, um "estar com" e "estar perto de", implicando consequentemente em uma relação com algo ou alguém (BRASIL, 2010).

No artigo de Sucigan, Toledo e Garcia (2012) que buscou compreender como os profissionais de enfermagem de equipes de saúde da família realizam o acolhimento dos 
pacientes de saúde mental, a prática de encaminhamentos como forma de tomada de decisão foi praticamente constante quando a demanda apresentada pelo usuário envolve questões relacionadas a saúde mental.

Os autores citados destacam, ainda, que a compreensão dos profissionais de enfermagem sobre o acolhimento é que este deve ser executado de forma rápida para que o maior número de usuários possa ser atendido e, neste contexto, os pacientes com queixas relacionadas à saúde mental devem ser encaminhados para equipes especializadas em saúde mental por compreenderem que o acolhimento a esse público necessita de tempo maior para ser realizado.

Entretanto, para além da falta de tempo necessário relatado pelos profissionais, a dificuldade em atender saúde mental, na perspectiva de cuidado, também é um fator influenciável para a realização de encaminhamentos para serviços especializados.

Aosani e Nunes (2013) investigando a percepção de trabalhadores da APS no tocante à saúde mental, evidenciaram que as dificuldades vão para além da conduta frente aos usuários com demandas de saúde mental, perpassando também para as dificuldades relacionadas à falta de profissionais, atuação em equipe, discussão de casos pertinentes, organização dos atendimentos, realização da prevenção, estabelecimento do vínculo terapêutico com o usuário e necessidade de qualificação e incentivo profissional.

O artigo de Sucigan, Toledo e Garcia (2012) ainda refere que as funções no acolhimento dos participantes estudados se caracterizavam por execução de técnicas e auxílio às práticas medicalizantes, limitando o acolhimento em distribuição de prescrições médicas refeitas e orientação sobre a posologia dos medicamentos prescritos.

No paradigma da medicalização enraizada na sociedade, o acolhimento se mostra como uma estratégia que retira a prioridade médica do atendimento, repassando a responsabilidade do cuidado para outros profissionais, em uma perspectiva de clinica ampliada, colocando o usuário frente a outras possibilidades de cuidado para além do medicamentoso (TESSER, 2010; ZANELLA et al., 2016).

Em contrapartida, o artigo de Mielke e Olschowsky (2011) buscou avaliar as tecnologias em saúde utilizadas nas ações de saúde mental por duas equipes de Estratégia Saúde da Família de uma Unidade de Saúde da Família. O estudo traz o acolhimento como promotor de saúde mental, mesmo que a acolhida ao usuário aparentemente não tenha sido resolutiva, pois entendem que o acolhimento implica em necessidade de saúde dos que procuram o serviço. O 
acolhimento em saúde mental aparece como importante ferramenta para tratamento e de reinserção social, por proporcionar também o estabelecimento de vínculo.

Os autores trazem que a escuta qualificada no momento do acolhimento como ferramenta essencial na acolhida em saúde mental, por dar voz ao sofrimento do usuário, minimizando o problema e facilitando a tomada de decisão.

Desta forma, Brasil (2010) afirma que este acolhimento é uma ferramenta essencial nos processos de trabalho em saúde que visa atender, de forma resolutiva e responsável, os usuários que procuram os serviços de saúde e, ressalta-se que esse modo de operar os processos de trabalho requer dos profissionais uma postura capaz de acolher, escutar e dar resposta mais adequada a demanda do usuário.

O encaminhamento como tomada de decisão no acolhimento não deve se restringir ao acolher e encaminhar, é preciso que o profissional seja responsável em orientar o usuário e a família em relação ao encaminhamento, sobre outros serviços de saúde para continuidade da assistência, como também garantir a eficácia do encaminhamento entrando em contato com o destino que o usuário está sendo encaminhado (BRASIL, 2010).

O artigo de Tanaka e Araújo (2012), corroborando com o estudo realizado por Mielke e Olschowsky (2011), buscou entender o acolhimento, considerando a percepção dos trabalhadores do Centro de Atenção Psicossocial e da Unidade Básica de Saúde da região Oeste de São Paulo. O estudo evidenciou que as práticas de acolhimento dos profissionais entrevistados estão distantes do que vem sendo sugerido para os serviços de saúde. Foi identificado nos entrevistados que a organização do processo de cuidar está voltada para as tecnologias duras.

Embora as tecnologias leves se apresentem fragilizadas no artigo supracitado, é evidente que elas são capazes de exercer mudanças na rede de cuidados trazendo o acolhimento, vínculo, responsabilização e visão integral do sujeito como subsídio para uma assistência de qualidade.

As tecnologias leves permitem a produção do cuidado baseado nas relações humanas, sendo a realização dos processos produtivos mediante a intercessão entre trabalhador e usuário por meio da escuta, do interesse, da construção de vínculo e confiança. São momentos construídos em ato que possibilitam captar a singularidade do sujeito em seu contexto social, cultural e universal, por assegurar ao usuário o seu momento de voz, de interagir e deixar sua marca no trabalhador (MERHY; FEUERWERKER, 2016). 
O acolhimento, como uma tecnologia leve, tem papel importante no processo de cuidar em saúde mental. Ele deve possibilitar um olhar ampliado do profissional para que o usuário não seja visto apenas como alguém que necessita de assistência em saúde, mas também, sobretudo, de necessidades biopsicossociais, econômicas, culturais e que seja visto como um sujeito único de direitos que deve ser percebido na sociedade.

Dessa forma, o acolhimento vai além do processo de triagem e encaminhamento, ele é transversal a toda a equipe do serviço, da rede de cuidados, do processo terapêutico do indivíduo (ANJOS, 2015).

\section{CATEGORIA 3: Barreiras encontradas no acolhimento em saúde mental na APS}

O artigo de Minóia e Minozzo (2015) traz a descrição de um processo de implantação de acolhimento às pessoas em sofrimento psíquico em uma unidade de APS. Durante o processo, os autores identificaram nos profissionais eventos/acontecimentos que se traduzem em: necessidade da conduta prescritiva, dificuldade de escuta dos aspectos subjetivos e prioridade para o que é físico.

Foi identificado nos profissionais dificuldade em conduzir o acolhimento, em saber manejar o que escutavam e de compreender a importância de fazer o usuário pensar e refletir seus problemas de saúde. Diante disso, as escapatórias dos profissionais voltavam-se para a conduta prescritiva no intuito de oferecer uma resposta imediata ao usuário.

No tocante à dificuldade de escuta dos aspectos subjetivos, ficou evidente que os profissionais quando se deparavam com o choro e sofrimento alheio, de imediato tentavam parar o choro, muitas vezes com utilização de medicamentos. Esse despreparo deixa visível o olhar para os aspectos biológicos do usuário e a angústia dos profissionais em lidar com a saúde mental.

No que se refere à prioridade para o que é físico, foi identificado que devido a equipe ser responsável por uma grande quantidade de usuários adscritos à unidade, o acompanhamento familiar e longitudinal torna-se difícil de ser realizado. Dessa forma, os profissionais acabam priorizando aspectos físicos dos usuários, deixando de lado demandas de saúde mental.

Essa visão prescritiva, medicalizante, produtora de procedimentos e consumidora de insumos tecnológicos potencializa a assistência médica hegemônica, restringindo a clínica na 
APS aos encaminhamentos para especialidades e realização de exames, que no âmbito da saúde mental fortalece a lógica manicomial (ARAÚJO; TANAKA, 2012).

Por outro lado, quando visto como dispositivo técnico-assistencial, o acolhimento permite uma reflexão sobre os modelos de atenção e gestão, de relações clínicas no trabalho e as relações de acesso aos serviços de saúde, sendo capaz de operar mudanças na assistência ao usuário, família e comunidade (BRASIL, 2010).

Nesse sentido, o acolhimento em saúde mental na APS possibilita a criação de vínculo entre os sujeitos envolvidos, permitindo o fortalecimento de laços e viabilizando ampliação na assistência ao usuário dos serviços de saúde.

\section{Considerações Finais}

A pesquisa evidenciou que o acolhimento em saúde mental na atenção primária à saúde é fundamental para uma assistência pautada na integralidade do cuidado, em especial ao usuário que sofre de problemas psíquicos, tornando-se imprescindível que os profissionais de saúde que trabalham em serviços de APS sejam capacitados para melhor acolher de forma eficiente os usuários

Os achados também apontam que a prática de encaminhamentos para serviços de saúde mental especializados, como o Centro de Atenção Psicossocial, é uma conduta tomada com frequência por alguns profissionais da APS. Essa decisão muitas vezes está intrínseca com a lógica manicomial e diretamente ligada com a dificuldade em atender usuários de saúde mental.

As equipes de saúde de APS devem ser motivadas para atuar frente a usuários com transtornos mentais, por estarem ligadas diretamente com o território, sendo então imprescindível capacitações para dar conhecimento e treinar os profissionais para à assistência a esse público.

As condutas prescritivas, medicalizantes, tecnicistas também são ações relatadas por alguns profissionais como formas de acolhimento, que erroneamente dão prioridade para as tecnologias duras e sintomas físicos apresentados pelos sujeitos, na perspectiva de parar o sofrimento de forma imediata. 
Assim, o acolhimento em saúde mental na atenção primária à saúde vem se apresentando de forma distinta da literatura, sendo caracterizado em um panorama de tecnologias duras na produção de saúde, centrado em especialidades, com mistura de modelos de atenção e pouco eficiente quanto à integralidade.

O acolhimento em saúde mental também aparece como uma das tecnologias leves que mais propicia a escuta qualificada e o estabelecimento de vínculo entre profissional e usuário, sendo então uma ferramenta indispensável para o tratamento do sujeito e sua reinserção social.

É necessário compreender o acolhimento como uma ferramenta que se efetiva no cotidiano das práticas de cuidado, devendo-se, sobretudo, ser resolutivo com a demanda do usuário, para que a qualidade da produção de saúde seja determinante no cuidado do sujeito.

\section{Referências}

ANJOS, M.A.; CARVALHO, P.A.L.; SENA, E.L.S. et al. Acolhimento da pessoa em sofrimento mental na atenção básica para além do encaminhamento. Cadernos Brasileiros de Saúde Mental. v.7, n.16, p. 27-40, 2015.

AOSANI, T.R.; NUNES, K.G. A Saúde Mental na Atenção Básica: A percepção dos Profissionais de Saúde. Revista Psicologia e Saúde. v.5, n.2, p. 71-80, 2013.

ARAUJO, A.K.; TANAKA, O.Y. Avaliação do processo de acolhimento em Saúde Mental na região centro-oeste do município de São Paulo: a relação entre CAPS e UBS em análise. Interface (Botucatu) [Internet]. v.16, n.43, p. 917-928, 2012. DOI: http://dx.doi.org/10.1590/S1414-32832012005000054.

BRASIL. Ministério da Saúde. Política Nacional de Atenção Básica. Portaria No 2.436, de 21 de setembro de 2017. Política Nacional de Atenção Básica; Brasília - DF. 2017.

BRASIL. Ministério da Saúde. Secretaria de Atenção à Saúde. Núcleo Técnico da Política Nacional de Humanização. Acolhimento nas práticas de produção de saúde. - 2. ed. 5. reimp. - Brasília: Editora do Ministério da Saúde, 2010.

CORREIA, V.R.; BARROS, S.; COLVERO, L.A. Saúde mental na atenção básica: prática da equipe de saúde da família. Rev. esc. enferm. USP. v.45, n.6, p. 1501-1506, 2011. DOI: http://dx.doi.org/10.1590/S0080-62342011000600032.

FILHO, J.A.S. Acolhimento Na Estratégia Saúde Da Família: Perspectiva Do Usuário (monografia). Centro de Ciências Biológicas e da Saúde: Universidade Regional do Cariri; 2016. 
GARCIA, M.I.H.; OLIVEIRA, A.M.N.; SEDREZ, J.P. et al. Realidade dos profissionais da estratégia de saúde da família em relação à detecção dos transtornos mentais comuns. VITTALLE-Revista de Ciências da Saúde. v.26, n.1, p. 37-44, 2014.

GIOVANELlA, L.; MENDONÇA, M.H.M. Atenção Primária à Saúde. In: GIOVANELLA, L. et al. (Org.). Políticas e Sistema de Saúde no Brasil. Editora Fiocruz; 2012. p.493-545.

JORGE, M.S.B.; PINTO, D.M.; QUINDERÉ, P.H.D. et al. Promoção da Saúde Mental Tecnologias do Cuidado: vínculo, acolhimento, co-responsabilização e autonomia. Ciênc. saúde coletiva. v.16, n.7, p. 3051-3060, 2011. DOI: http://dx.doi.org/10.1590/S141381232011000800005 .

MENDES, K.D.S.; SILVEIRA, R.C.C.P.; GALVÃO, C.M. Revisão integrativa: método de pesquisa para a incorporação de evidências na saúde e na enfermagem. Texto Contexto Enferm. v.17, n.4, p.758-764. 2008. DOI: http://dx.doi.org/10.1590/S0104-07072008000400018.

MERHY, E.E.; FEUERWERKER, L.C.M. Novo olhar sobre as tecnologias de saúde: uma necessidade contemporânea. In: Mandarino ACS, Gomberg E (Org.). Leituras de novas tecnologias e saúde. São Cristóvão: Editora UFS, 2009. p. 29-74.

MIELKE, F.B.; OLSCHOWSKY, A. Ações de saúde mental na estratégia saúde da família e as tecnologias em saúde. Esc. Anna Nery [Internet]. v.15, n.4, p. 762-768, 2011. DOI: http://dx.doi.org/10.1590/S1414-81452011000400015.

MINÓIA, N.P.; MINOZZO, F. Acolhimento em Saúde Mental: Operando Mudanças na Atenção Primária à Saúde. Psicol., Ciênc. Prof. v.35, n.4, p.1340-1349, 2015. DOI: https://dx.doi.org/10.1590/1982-3703001782013.

RODRIGUES, J.; BROGNOLI, F. Acolhimento no serviço de atenção psicossocial. Cadernos Brasileiros de Saúde Mental. v.6, n.13, p.61-74, 2014.

RODRIGUES, L.B.B; SILVA, P.C.S; PERUHYPE, R.C. et al. A atenção primária à saúde na coordenação das redes de atenção: uma revisão integrativa. Ciênc. saúde coletiva [Internet]. v.19, n.2, p.343-352, 2014. DOI: http://dx.doi.org/10.1590/1413-81232014192.18032012.

SANTOS, I.M.V.; SANTOS, A.M. Acolhimento no Programa Saúde da Família: revisão das abordagens em periódicos brasileiros. Revista de Salud Pública. v.13, n.4, p.703-716, 2011.

SCHEIBEL, A.; FERREIRA, L.H. Acolhimento no CAPS: reflexões acerca da assistência em saúde mental. Rev. Baiana de Saúde Pública. v.35, n.4, p. 966-983, 2011.

SILVA, T.F.; ROMANO, V.F. Sobre o acolhimento: discurso e prática em Unidades Básicas de Saúde do município do Rio de Janeiro. Saúde debate. v.39, n.105, p; 363-374, 2015. DOI: http://dx.doi.org/10.1590/0103-110420151050002005. 
SOARES, A.C.; TRINDADE, L.; RODRIGUES, G.C.S. et al. Análise Clínica-Epidemiológica De Pacientes Portadores De Transtorno Mental Na Amazônia Brasileira. Rev. Bras. Neurol. Psiquiatr. v.19, n.2, p.96-107, 2015.

SOARES, C.B.; HOGA, L.A.K.; PEDUZZI, M. et al. Revisão integrativa: conceitos e métodos utilizados na enfermagem. Rev. esc. enferm. USP [Internet]. v.48, n.2, p. 335-345, 2014. DOI: http://dx.doi.org/10.1590/S0080-6234201400002000020.

SUCIGAN, D.H.I.; TOLEDO, V.P.; GARCIA, A.P.R.F. Acolhimento E Saúde Mental: Desafio Profissional Na Estratégia Saúde Da Família. Rev Rene. v.13, n.1, p. 2-10, 2012. DOI: http://dx.doi.org/10.15253/rev\%20rene.v13i1.3756.

TESSER, C.D.; NETO, P.P.; CAMPOS, G.W.S. Acolhimento e (des)medicalização social: um desafio para as equipes de saúde da família. Ciênc. saúde coletiva. v.15, supl. 3, p. 3615-3624, 2010. DOI: http://dx.doi.org/10.1590/S1413-81232010000900036.

WORLD HEALTH ORGANIZATION. The World Health Report - 2001: Mental Health: New understanding. New Hope; 2001. Disponível em: www.who.int/en.

\section{Como citar este artigo (Formato ABNT):}

SILVA FILHO, José Adelmo da; BEZERRA, Adriana de M. Acolhimento em Saúde Mental na Atenção Primária à Saúde: Revisão Integrativa. Id on Line Rev.Mult. Psic., 2018, vol.12, n.40, p.613-627. ISSN: 1981-1179.

Recebido: 07/05/2018

Aceito 10/05/2018 